\title{
Special section on image and video processing for security
}

\author{
Sudha Natarajan · Niladri Bihari Puhan · Pramod \\ Kumar Meher
}

Published online: 16 April 2013

(c) Springer-Verlag London 2013

Research on personal and public information security has been of great emphasis in recent years. The ubiquitous availability of digital cameras, ranging from surveillance systems to mobile phones, makes image and video contents as predominant form of information used for the analysis in security applications.

Video surveillance is the most common way to monitor and enhance the security at strategic locations. Automated surveillance systems, which replace the current manual surveillance, require efficient pattern recognition for object detection, tracking, and suspicious activity identification. Another way of enforcing security, especially at immigration and check points, is identification of people based on the physical appearance or behavioral characteristics. New image- and video-based biometrics are evolved over the years to improve the accuracy of identification for strengthening the security.

When visual data is abundant, their reliability will be questioned due to the ease in counterfeiting the content. Digital image/video forensics is an emerging research field which aims at validating the authenticity of images by detecting traces of forgeries. Furthermore, secure transmission of images and video over network without tampering is achieved through cryptography and steganography. These are widely used techniques that manipulate data by encrypting or hiding information.

\footnotetext{
N. Sudha $(\varangle)$

XMOS Semiconductor India Pvt. Ltd., Chennai, India

e-mail: sudhanatraj@yahoo.com

N. B. Puhan

Indian Institute of Technology, Bhubaneswar, India

P. K. Meher

Institute of Infocomm Research, Singapore, Singapore
}

This special section addresses the latest developments in the image and video processing technologies for security applications.

The first three papers present new techniques in various biometrics. Cho presents a new method based on generalized neural reflectance model for enhancing ear recognition under varying illumination. The recognition algorithm in this paper uses a similarity measure on auditory features extracted through principal component analysis and then further processed by Fisher's discriminant analysis to acquire lower-dimensional patterns. The experimental results provided in this paper show that lower error rates are achieved under different variations in lighting. The second paper by Zhou et al. presents a comprehensive multimodal eye recognition system based on both iris and sclera patterns for recognition. They achieve better recognition accuracy than independent recognition of iris or sclera patterns. In addition, they propose a multimodal multi-angle eye recognition system that further improves the recognition accuracy. In the third paper, Hegde et al. introduce a novel biometric trait, namely finger knuckle print. They present three different algorithms for authentication and evaluated their performance.

The next three papers are in the field of data security. Karthik and Kashyap present a transparent privacy preserving hashing scheme tailored to preserve the DCT AC coefficient distributions, despite a constrained inter-block shuffling operation. These DCT distributions are mapped onto a generalized Gaussian model characterized by shape and scale parameters, quantized and gray coded into a binary hash matrix. They have shown that their encryption scheme is perceptually secure and does not impair the search reliability and accuracy of the hashing procedure. In the next paper, Dutta et al. present a technique for content-based video copy detection. In their technique, fingerprint is generated from the sub-bands obtained by wavelet decomposition intensity 
image. The effectiveness of their technique is established through experiments. The third paper in this area is presented by Shahid et al. They propose a novel approach for active fingerprinting of state-of-the-art video codec H.264/AVC. The robustness of their approach is proved by performing linear and nonlinear collusion attacks in the pixel domain.

The last two papers are closely related to video surveillance. Tang et al. propose an efficient method of cast shadow removal for video sequences with static background using multiple features.The idea behind their method is that a simultaneous use of multiple features reduces the similarity between object and shadow pixels while most existing methods depend on this similarity. Their experimental results demonstrate the superiority of their method over other methods. In the next paper, Tu and Bhattacharya present a gametheoretic approach for coordinated multi-resolution tracking over arbitrary floor plan. They have proposed an enhanced radial sweep algorithm to find the polygon of visibility at any point on or inside a polygon that contains vision-obstructing polygonal entities. 